\title{
LYCOPENEMIA IN A PATIENT WITH THALASSEMIA MINOR
}

\author{
Vessela Raykova \\ Department of Medical Microbiology, Faculty of Medicine, Medical University - Sofia
}

\begin{abstract}
An 18-year-old girl presented with yellowish discoloration of her palms that had appeared several days earlier. The rest of her skin, mucous membranes and sclera were with normal color. She denied history of anorexia, jaundice, pruritus, loss of appetite, nausea, vomiting, abdominal pain or change in the color of her urine or stool or any other symptom. She reported the presence of thalassemia minor and increased beet consumption $(0.5 \mathrm{~kg} / \mathrm{day})$ in the last one and a half month. After conducting the necessary research tests anorexia, hypothyroidism, renal failure and diabetes mellitus were ruled out. The discoloration was diagnosed as lycopenemia. Simple changes in diet brought complete resolution of the symptoms.
\end{abstract}

Keywords: yellow palms, lycopenemia, carotenemia

\section{INTRODUCTION}

Lycopenemia (a variant of carotenemia) is a benign clinical entity caused by excessive ingestion of foods containing lycopene characterized by yellowish orange pigmentation of the skin $(1,2)$ It is caused by the deposition of lycopene. Lycopene is a structural isomer of $\beta$-carotene and an antioxidant. It could be found in different products as tomatoes, rosehips, chili beans, beets (3). The lycopene deposits occur predominantly in stratum corneum of the skin, which has a high lipid content and hence an affinity for lycopene (4). A patient with yellowish discoloration of the palms with thalassemia minor and dietary deviations is the focus of this case report (Fig. $1,2)$.

Address for correspondence:

Vessela Raykova

Faculty of Medicine

Medical University-Sofia

15 Akademik Ivan Geshov Blvd

1431 Sofia

e-mail:pumpi@abv.bg

Received: May 2, 2019

Accepted: June 17, 2019

\section{DISCUSSION}

An 18-year-old girl presented in the hospital with yellowish discoloration of her palms that had appeared several days earlier. No pain, no itching or any other complaints were reported. On the clinical study the rest of her skin, mucous membranes, in-

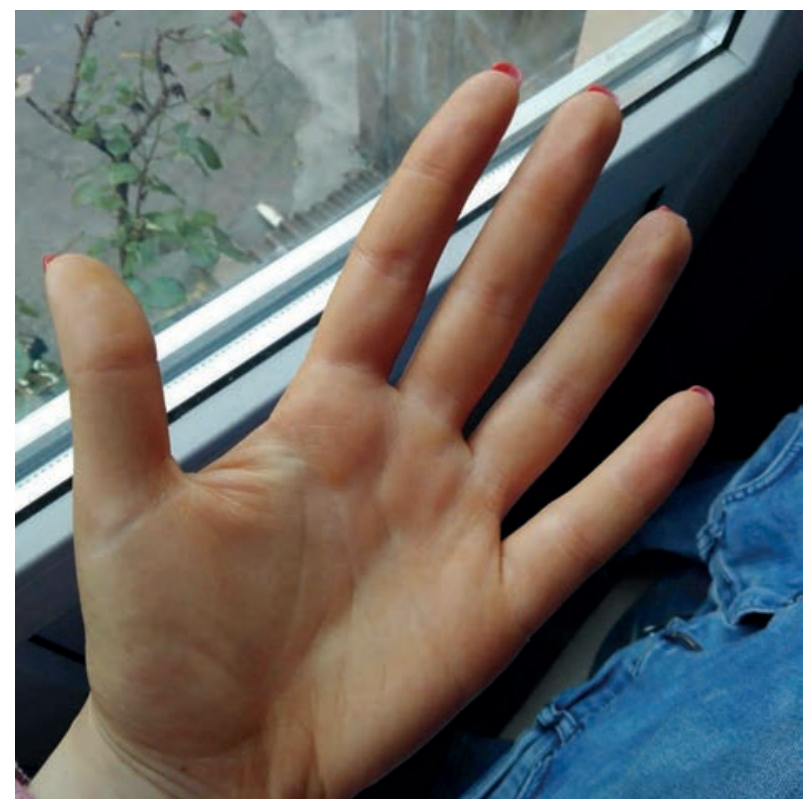

Fig. 1. Yellowish discoloration of patient's palm 


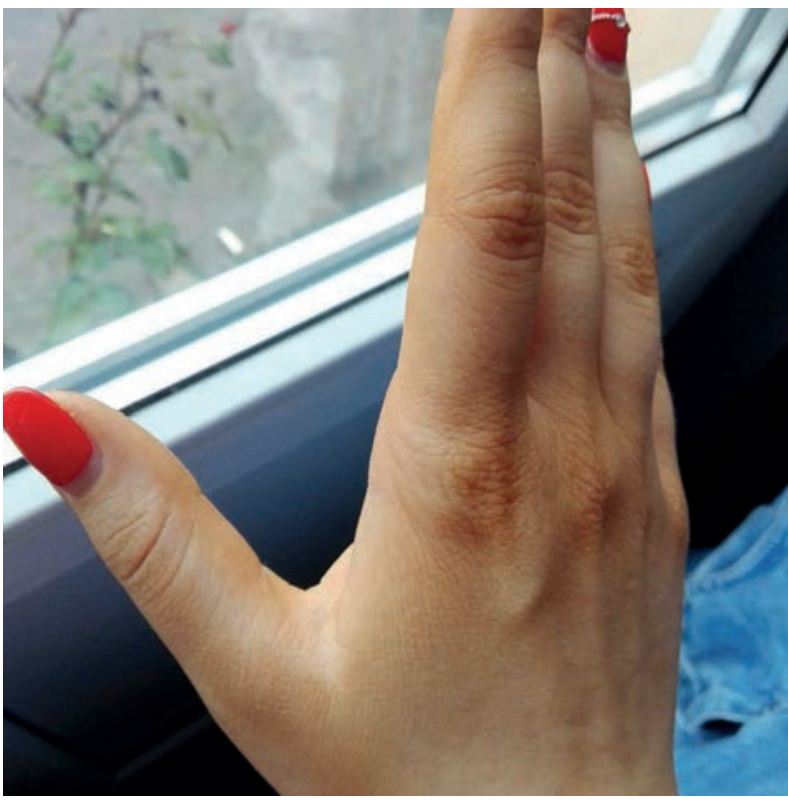

Fig. 2. Yellowish discoloration of patient's back of palm

cluding sclera, were with normal color. The patient denied history of recent infectious disease, anorexia, jaundice, pruritus, loss of appetite, nausea, vomiting, abdominal pain or change in the color of her urine or stool or any other symptom. As accompanying state the girl reported presence of thalassemia minor. And the detailed patient's history detected deviations in the diet - increased beet consumption $(0.5 \mathrm{~kg} /$ day) in the last one and a half month. We present to your attention this clinical case as interesting in a differential diagnostic plan.

The yellow hands are not a disease in themselves but a symptom of an underlying condition responsible for the change of color in hands and sometimes in other parts of the body. Many internal conditions can cause yellowing of the skin - ingestion of nutritional supplements, anorexia, hypothyroidism, renal and hepatic failure, diabetes mellitus. So, the discoloration could be something benign or a more serious condition that requires specialized medical intervention; hence the importance of an adequate clinical diagnosis, because a wrong approach can have serious consequences for the patient.

Due to the yellowish discoloration of the palms of our patient and no change in the color of other parts of the skin or mucous membranes, together with the lack of history of anorexia, jaundice, pruritus, loss of appetite, nausea, vomiting, abdominal pain, change in the color of urine or stool or any other symptom, we chose a working diagnosis of carotenemia and more precisely lycopenemia.

Carotenemia is the medical terminology describing yellow-orange skin pigmentation due to high levels of carotene in blood (5). Excessive consumption of fruits and/or vegetables high in carotene content is often the culprit (6). Carotenoids are organic compounds found in various plants and food items - apricot, cantaloupe, mango, orange, papaya, peaches, prunes, carrots, beet, green beans, asparagus, broccoli, cucumber, lettuce, parsley, spinach, squash, mustard, pumpkins, kale, and sweet potatoes. Beta-Carotene is the main carotenoid found in plants and is most responsible for this condition (7). Excessive ingestion (greater than $30 \mathrm{mg}$ a day) for a prolonged period could lead to carotenemia (8).

The lycopenemia - a variant of carotenemia, accepted as a diagnosis in our patient, is a benign and completely harmless condition. The condition arises as a result of excess levels of $\beta$-carotene in the body. In most cases, lycopenemia can be diagnosed on the basis of patient's dietary history but surely specific tests are indicated to rule out other possible reasons for the condition (9). In our patient there was a history of increased beet consumption and lycopenemia could be accepted as a reason for the yellowish discoloration of her palms.

The presence of thalassemia minor in our patient required a thorough examination of blood and liver function as well. Thalassemia is an inherited blood disorder in which the body makes an abnormal form of hemoglobin. The disorder results in excessive destruction of red blood cells, which leads to anemia and symptoms as bone deformities (especially in the face), dark urine, delayed growth and development, excessive tiredness and fatigue, and yellow or pale skin. After conducting the necessary research tests it was decided that thalassemia in this case did not affect the change in the color of the skin. Of course all the other conditions that could cause yellowing of the skin -anorexia, hypothyroidism, renal failure and diabetes mellitus were ruled out. The levels of our patient's liver enzymes, $\alpha$-carotene, vitamin A, lutein, zeaxanthin, thyroid-stimulating hormone, blood sugar and glycosylated hemoglobin were normal. 
Lycopenemia is a condition which does not require any specific treatment and could be managed with diet alone (10). So, our patient was advised to reduce her beet consumption and to apply hand cream with urea $12 \%$ twice per day for 1-2 weeks. The color of her palms gradually returned to normal in 3-4 weeks.

\section{REFERENCES}

1. Jawaid AS, Koti M. Orange discoloration of the palms. Can Med Assoc J. 2009; 180(8):895. doi:10.1503/cmaj.071335.

2. James W, Berger T, Elston D. Andrews' Diseases of the Skin: Clinical Dermatology. 10th ed. Saunders; 2005.

3. McConaghey RM. Carotenaemia. Lancet. 1952; 2(6737):714-5. doi: 10.1016/s0140-6736(52)91324-x.

4. Hughes JD, Wooten RL. The orange people. J Am Med Assoc. 1966; 197(9):730-1.
5. Arya V, Grzybowski J, Schwartz RA. Carotenemia. Cutis. 2003; 71(6):441-2, 448.

6. Leung AK. Benign carotenemia in children. Can Fam Physician. 1989; 35:81-3.

7. Cohen. Observations on carotenemia. Ann. Intern. Med. 1958; 48(2):219-27.

8. Julka S, Jamdagni N, Verma S, Goyal R. Yellow palms and soles: A rare skin manifestation in diabetes mellitus. Indian J Endocrinol Metab. 2013; 17(Suppl 1):S299-300. doi: 10.4103/2230-8210.119625.

9. Chhabra P, Bhasin DK. Image Diagnosis: Yellow palms and soles: Look beyond the eyes and think beyond hyperbilirubinemia. Perm J. 2017; 21:17034. doi: 10.7812/TPP/17-034.

10. Kaimal S, Thappa DM. Diet in dermatology: revisited. Indian J Derm Venereol Leprol. 2010; 76(2):103-15. doi: 10.4103/0378-6323.60540. 